PROCEEDINGS OF THE

AMERICAN MATHEMATICAL SOCIETY

Volume 128, Number 1, Pages 255-260

S 0002-9939(99)04958-8

Article electronically published on May 6, 1999

\title{
AN INFINITE FAMILY OF MANIFOLDS WITH BOUNDED TOTAL CURVATURE
}

\author{
A. N. DRANISHNIKOV
}

(Communicated by James E. West)

\begin{abstract}
The negative answer to the following problem of V. I. Arnold is given: Is the number of topologically different $k$-manifolds of bounded total curvature finite?
\end{abstract}

\section{$\S 1$. INTRODUCTION}

In $[\mathrm{Ar}] \mathrm{V}$. I. Arnold defined the total curvature $\alpha(g)$ of an immersion $g$ : $M^{k} \rightarrow \mathbf{R}^{m}$ of a $k$-manifold in the $m$-dimensional euclidean space as the $k$-volume $\operatorname{vol}_{k}(g \Delta \bar{g})\left(M^{k}\right)$ of a submanifold $(g \Delta \bar{g})\left(M^{k}\right) \subset \mathbf{R}^{m} \times G_{m, k}$ where $\bar{g}: M^{k} \rightarrow G_{m, k}$ is the induced map to the Grassmann manifold. He proved that for any two submanifolds $X, Y \subset \mathbf{R}^{m}$ and for every diffeomorphism $A: \mathbf{R}^{m} \rightarrow \mathbf{R}^{m}$ generically the total curvature $\alpha\left(A^{n}(X) \cap Y\right)$ is bounded by $C e^{\lambda n}$ where $A^{n}=A \circ \ldots \circ A$ and $C$ and $\lambda$ do not depend on $n$. Then the following question of Arnold appeared naturally: Is the number of topologically different $k$-manifolds $M$ lying in $\mathbf{R}^{m}$ with the total curvature $\alpha(M)$ bounded from above finite?

We note that Arnold's total curvature $\alpha(g)$ differs from the classic Chern-Lashof total curvature $\tau(g)[\mathrm{N}-\mathrm{K}]$. Recall that $\tau(g)=\frac{1}{\operatorname{vol}\left(S^{m-1}\right)} \int_{S M^{k}} \nu^{*}(d \sigma)$ where $S M^{k}$ is the unit sphere bundle of the normal bundle of an immersion $g: M^{k} \rightarrow \mathbf{R}^{m}$, $\nu: S M^{k} \rightarrow S^{m-1}$ is the Gauss map and $d \sigma$ is the volume element on $S^{m-1}$. Arnold's total curvature is always greater than Chern-Lashof's and the last can be estimated from below by the Morse number $m\left(M^{k}\right)=$ the minimal number of critical points of Morse functions.

It is easy to show that the answer to Arnold's question is affirmative for 2manifolds. The purpose of this paper is to show that for 3-manifolds the answer is negative.

Theorem 1. There exist a number $C>0$ and an infinite family $\left\{M_{i}\right\}$ of pairwise nonhomeomorphic 3-dimensional compact submanifolds of $\mathbf{R}^{4}$ such that the total curvature $\alpha\left(M_{i}\right)<C$ for all $i$.

Received by the editors December 26, 1992 and, in revised form, March 24, 1998

1991 Mathematics Subject Classification. Primary 53C22; Secondary 53C42, 57C42.

Key words and phrases. Total curvature, immersion, Casson invariant, Dehn surgery, Seifert manifold.

The author was partially supported by NSF grant DMS-9500875.

(C)1999 American Mathematical Society 
Theorem 2. There exist a number $C>0$ and an infinite family $\left\{\Sigma_{i}\right\}$ of pairwise nonhomeomorphic homology 3-spheres with embeddings $\left\{\eta_{i}: \Sigma_{i} \rightarrow \mathbf{R}^{7}\right\}$ such that $\alpha\left(\eta_{i}\right)<C$ for all $i$.

The construction of both families of manifolds is basically due to the presence of the fundamental group. This situation suggests the following:

Conjecture. The answer to Arnold's problem is positive for simply connected manifolds.

\section{$\S 2$. The CONSTRUCTION OF THE FIRST FAMILY}

Let $K(n, m) \subset S^{3}$ be an $(n, m)$-torus knot in $S^{3}$ and let $N(n, m)$ be its regular neighborhood. Let $\left\{p_{i}\right\}$ be a sequence of all odd numbers. For every $i$ let $M_{i}$ denote the result of doubling the compact 3 -manifold $S^{3}-\operatorname{Int} N\left(2, p_{i}\right)$ along its boundary $\partial N\left(2, p_{i}\right)$.

Lemma 1. For every $i \neq j$ manifolds $M_{i}$ and $M_{j}$ are not homeomorphic.

Proof. An $S^{1}$-action on $S^{3}=S^{1} * S^{1}$ defined by the formula

$$
(\theta,(\phi, \psi)) \rightarrow\left(\phi+2 \theta, \psi+p_{i} \theta\right)
$$

has two exceptional orbits with the orbit space homeomorphic to $S^{2}$. We may assume that $N\left(2, p_{i}\right)$ is a preimage of a 2 -disk under the projection to the orbit space. Then it easy to see that each $M_{i}$ is a Seifert manifold [Or] with the orbit space $S^{2}$ with 4 exceptional orbits of types $(2,1),(2,1),\left(p_{i}, 2\right),\left(p_{i}, 2\right)$. Since these orbit invariants are different for $i \neq j$, Seifert bundles on $M_{i}$ and $M_{j}$ are different. Note that $M_{i}$ are large Seifert manifolds for all $i$. Hence $M_{i}$ and $M_{j}$ are not homeomorphic for $i \neq j$ [Or, Theorem 6].

Lemma 2. Suppose that $g_{i}: M \rightarrow N$ is a sequence of embeddings of a Riemannian manifold $M$ into a Riemannian manifold $N$, converging in the $C^{1}$-topology to an embedding $g: M \rightarrow N$. Then $\lim \operatorname{vol}\left(g_{i}(M)\right)=\operatorname{vol}(g(M))$, where $\operatorname{vol}(L)$ for $L \subset N$ means the volume of submanifold $L$ in $N$.

The proof follows for instance from [D-N-F], v.1.

Let $D^{m}$ denote the unit ball in $\mathbf{R}^{m}$ and let $D_{r}^{m}$ be a concentric ball of radius $r$. The normal regular neighborhood of radius $r$ of immersed $k$-manifold $f: M^{k} \rightarrow \mathbf{R}^{m}$ is an immersion $F: M^{k} \times D_{r}^{m-k} \rightarrow \mathbf{R}^{m}$ such that 1) $\left.F\right|_{M^{k} \times\{0\}}=f$ where 0 is the center of the disk $\left.D_{r}^{m-k}, 2\right)$ for every $t \in M^{k}$ the restriction $\left.F\right|_{\{t\} \times D_{r}^{n-k}}$ is an isometrical embedding and the disk $F\left(\{t\} \times D_{r}^{m-k}\right)$ is orthogonal to the manifold $f\left(M^{k}\right)$ at the point $f(t)$.

Every $C^{1}$-manifold has locally a normal regular neighborhood for some $r[\mathrm{H}]$, and hence, every closed manifold with trivial normal bundle has a normal regular neighborhood. Therefore every smooth enough immersion of the circle in $\mathbf{R}^{3}$ has a normal regular neighborhood. Here is a parameterized version of that statement.

Lemma 3. Suppose that $\left\{f_{i}: S^{1} \rightarrow \mathbf{R}^{3}\right\}$ is a sequence of immersions of $S^{1}$ into $\mathbf{R}^{3}$, converging in $C^{2}$-metric to an immersion $f_{\infty}$. Then there exist $r>0$ and normal regular neighborhoods $F_{i}: S^{1} \times D_{r} \rightarrow \mathbf{R}^{3}, i=1,2, \ldots, \infty$, such that $\left\{F_{i}\right\}$ converges in $C^{2}$-metric to $F_{\infty}$. 
Let $C_{k}($,$) be a space of mappings in C^{k}$-topology. Denote by $S^{1}$ the boundary of the unit disk $D^{2}$ and denote by $w_{r}: S^{1} \rightarrow \partial D_{r}$ the natural projection. In this notation the center of $D^{2}$ is $\partial D_{0}$. Suppose that $f: S^{1} \rightarrow \mathbf{R}^{3}$ is a $C^{2}$-immersion with a normal regular neighborhood $F$ of radius $r$. We define a map $R_{f}:[0, r] \rightarrow$ $C_{2}\left(S^{1} \times S^{1}, \mathbf{R}^{3}\right)$ by setting $R_{f}(x)=F \circ\left(i d_{S^{1}} \times w_{r}\right): S^{1} \times S^{1} \rightarrow \mathbf{R}^{3}$.

Lemma 4. Suppose that a sequence $\left\{f_{i}: S^{1} \rightarrow \mathbf{R}^{3}\right\}$ is converging to $f_{\infty}$ in $C^{2}$ metric and assume that $r$ is given by Lemma 3. Then $R_{f_{i}}$ uniformly converges to $R_{f_{\infty}}$.

We omit the proof since it is straightforward.

We define the 'Gauss map' $G_{f}:[0, r] \rightarrow C_{1}\left(S^{1} \times S^{1}, G_{3,2}\right)$ for $R_{f}$ in the following way. For $x>0$ the value $G_{f}(x)$ is the tangent bundle map $\nu: S^{1} \times S^{1} \rightarrow G_{3,2}$ for $S^{1} \times \partial D_{x}$. For $x=0$ for every $(t, \theta) \in S^{1} \times S^{1}$ the value $G_{f}(0)(t, \theta)$ is the span of the derivative $f^{\prime}(t)$ and the vector orthogonal to $F(t,(r, \theta))-F(t, 0)=b(t, \theta)$. Here $(r, \theta) \in D_{r}$ means a point written in polar coordinates. Note that for all $x \in[0, r]$ the Gauss map is defined as $G_{f}(x)(t, \theta)=\operatorname{span}\left\{\frac{\partial F(t,(x, \theta))}{\partial t}, b(t, \theta)^{\perp}\right\}$.

Lemma 5. Suppose that a sequence $\left\{f_{i}: S^{1} \rightarrow \mathbf{R}^{3}\right\}$ is converging to $f_{\infty}$ in $C^{2}$ metric and assume that the number $r$ is provided by Lemma 3. Then $G_{f_{i}}$ converges uniformly to $G_{f_{\infty}}$.

Proof. According to Lemma 3 a sequence $F_{i}$ converges uniformly to $F_{\infty}$ in $C^{2}$ metric. It implies a uniform convergence of $\frac{\partial F_{i}}{\partial t}$ to $\frac{\partial F_{\infty}}{\partial t}$ in $C^{1}$-metric. The second vector $b_{i}(t, \theta)^{\perp}$ does not depend on $x$, and therefore by virtue of Lemma 3 the sequence $b_{i}(t, \theta)^{\perp}$ converges to $b_{\infty}(t, \theta)^{\perp}$ in $C^{1}$-metric as a sequence of functions in $t$ and $\theta$. This implies the lemma.

The proof of Theorem 1. We construct manifolds $M_{i}$ in $\mathbf{R}^{4}$ symmetrically with respect to the hyperplane $\{0\} \times \mathbf{R}^{3}$. Let us consider two symmetric 3-dimensional spheres $S_{+}^{3} \subset[1, \infty) \times \mathbf{R}^{3}$ and $S_{-}^{3} \subset(-\infty,-1] \times \mathbf{R}^{3}$ such that the intersection $S_{+}^{3} \cap\left(\{1\} \times \mathbf{R}^{3}\right)$ is a 3 -disk $D_{+}^{3}$ and similarly $S_{-}^{3} \cap\left(\{-1\} \times \mathbf{R}^{3}\right)=D_{-}^{3}$. Let $S^{1} \subset D_{+}^{3}$ be a smoothly embedded circle and let $f: S^{1} \rightarrow S^{1} \subset D_{+}^{3}$ be a double winding around that circle $\left(f \in C^{2}\right)$. For every $i$ we consider the boundary of the normal regular neighborhood of $S^{1}$ of radius $\epsilon_{i}$ and realize a torus knot $K\left(2, p_{i}\right)$ on it. For small enough $\epsilon_{i}$ it is possible to realize the knot $K\left(2, p_{i}\right)$ by a map $f: S^{1} \rightarrow \mathbf{R}^{3}$ which is $1 / i$-close to $f$ in $C^{2}$-metric. By virtue of Lemma 3 there exist a number $r$ and the normal regular neighborhoods $F_{i}$ of radius $r$. For every $i$ there exists $r_{i}<r$ such that the restriction of $F_{i}$ on $S^{1} \times D_{r_{i}}^{2}$ is an embedding. We denote $N\left(2, p_{i}\right)=F_{i}\left(S^{1} \times D_{r_{i}}^{2}\right)$. Let $C_{i}$ be the cylinder over the boundary $\partial N\left(2, p_{i}\right)$ that connects $S_{+}^{3}$ with $S_{-}^{3}$. We define $M_{i}=\left(S_{+}^{3}-\operatorname{Int} N\left(2, p_{i}\right)\right) \cup C_{i} \cup\left(S_{-}^{3}-\operatorname{Int} N\left(2, p_{i}\right)\right)$.

We will write $\alpha(M)$ if the immersion of $M$ is fixed. Since

$$
\alpha\left(M_{i}\right) \leq \alpha\left(S_{+}^{3}\right)+\alpha\left(S_{-}^{3}\right)+\alpha\left(\partial N\left(2, p_{i}\right) \times[-1,1]\right),
$$

it suffices to find a common estimate for $\alpha\left(\partial N\left(2, p_{i}\right) \times[-1,1]\right)$. By an obvious version of the Kuiper theorem $[\mathrm{N}-\mathrm{K}]$ for the Arnold total curvature it follows that $\alpha\left(\partial N\left(2, p_{i}\right) \times[-1,1]\right)=2 \alpha\left(\partial N\left(2, p_{i}\right)\right)$.

Lemmas 4, 5 imply that $\lim _{i \rightarrow \infty} R_{f_{i}}\left(r_{i}\right)=R_{f_{\infty}}(0)$ and $\lim _{i \rightarrow \infty} G_{f_{i}}\left(r_{i}\right)=G_{f_{\infty}}(0)$. Denote by $A_{i}$ the diagonal product of $R_{i}$ and $G_{i}, i=1,2, \ldots, \infty$. By Lemma 2 it follows that $\lim _{i \rightarrow \infty} \operatorname{vol}\left(A_{i}\left(r_{i}\right)\left(S^{1} \times S^{1}\right)\right)=\operatorname{vol}\left(A_{\infty}(0)\left(S^{1} \times S^{1}\right)\right)$. Since $\operatorname{vol}\left(A_{i}\left(r_{i}\right)\left(S^{1} \times S^{1}\right)\right)=\alpha\left(\partial N\left(2, p_{i}\right)\right)$, it follows that the sequence $\alpha\left(\partial N\left(2, p_{i}\right)\right)$ 


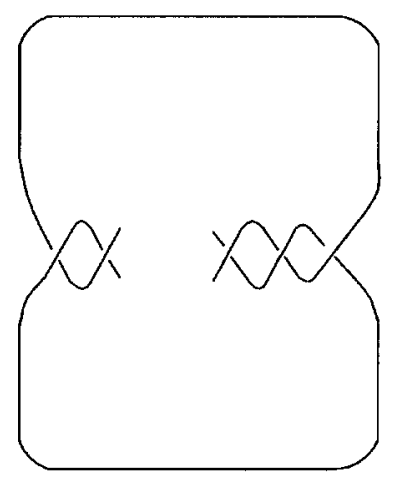

Figure 1

has an upper bound. So, it remains to note that by Lemma 1 the family $\left\{M_{i}\right\}$ is pairwise nonhomeomorphic.

Remark. Manifolds $M_{i}$ are not smoothly embedded in $\mathbf{R}^{4}$ because at the points where spheres $S_{+}^{3}$ and $S_{-}^{3}$ are attached to the cylinder $C_{i}$ we have singularities. If we smooth these angles by a small perturbation near $\partial N\left(2, p_{i}\right)$, then $\alpha\left(M_{i}\right)$ will increase by approximately $\operatorname{vol}\left(\partial N\left(2, p_{i}\right)\right) \times \pi / 2$. Since $\operatorname{vol}\left(\partial N\left(2, p_{i}\right)\right) \rightarrow 0$, then the sequence $\alpha\left(M_{i}\right)$ for resulting smooth manifolds $M_{i}$ also will be bounded.

\section{$\S 3$. The COnstruction of The SECOND FAmily}

For any knot $K \subset S^{3}$ we denote by $K_{1}$ the 3 -manifold obtained from $S^{3}$ by Dehn surgery on the knot $K$ with the homology class $(1,1)[\mathrm{R}]$. Let $K^{m}$ be the following knot with $2 m+1$-crossings shown in Figure 1:

Lemma 6. The manifolds $K_{1}^{m}$ and $K_{1}^{n}$ are homeomorphic if and only if $m=n$.

Proof. The direct computation of the Casson invariant $\lambda\left(K_{1}^{k}\right)$ shows that $\lambda\left(K_{1}^{m}\right)$ and $\lambda\left(K_{1}^{n}\right)$ are different for $m \neq n[\mathrm{~A}-\mathrm{M}]$.

By the definition a normal regular neighborhood induces a framing of the normal vector bundle. Let $\xi: S^{1} \rightarrow \mathbf{R}^{3}$ be an immersion of the circle in $\mathbf{R}^{3}$ and let $F^{k}: S^{1} \times D_{r}^{2} \rightarrow \mathbf{R}^{3}$ be a normal regular neighborhood of $\xi$ of radius $r$ such that the induced framing has Hopf invariant equal to $k$. We consider the embedding of $\mathbf{R}^{3}$ in $\mathbf{R}^{7}$ as a factor $\mathbf{R}^{3} \times 0 \times \ldots \times 0 \subset \mathbf{R}^{7}$. A map $\phi:(M, N) \rightarrow\left(\mathbf{R}^{7}, \mathbf{R}^{3}\right)$ is said to be a relative embedding if the restriction of $\phi$ on $N$ is an immersion into $\mathbf{R}^{3}$ and the restriction of $\phi$ on $M-N$ is an embedding in $\mathbf{R}^{7}-\mathbf{R}^{3}$, transversal to $\mathbf{R}^{3}$.

Lemma 7. Let $f:\left(D^{2}, \partial D^{2}\right) \rightarrow\left(\mathbf{R}^{7}, \mathbf{R}^{3}\right)$ be a relative embedding of class $C^{2}$. Then there exist $r>0$ and a relative embedding of class $C^{2}, F:\left(D^{2} \times D_{r}^{2}, \partial D^{2} \times\right.$ $\left.D_{r}^{2}\right) \rightarrow\left(\mathbf{R}^{7}, \mathbf{R}^{3}\right)$, such that

1) $\left.F\right|_{D^{2} \times\{0\}}=f$.

2) $\left.f\right|_{\partial D^{2} \times D_{r}^{2}}=F^{1}$ is a normal regular neighborhood in $\mathbf{R}^{3}$, with Hopf invariant equal to one.

3) For every $x \in$ Int $D^{2}$ the restriction $\left.F\right|_{\{x\} \times D_{r}^{2}}$ imbeds the disk $D_{r}^{2}$ isometrically into the 5-plane, orthogonal to $f\left(D^{2}\right)$ at the point $f(x)$. 


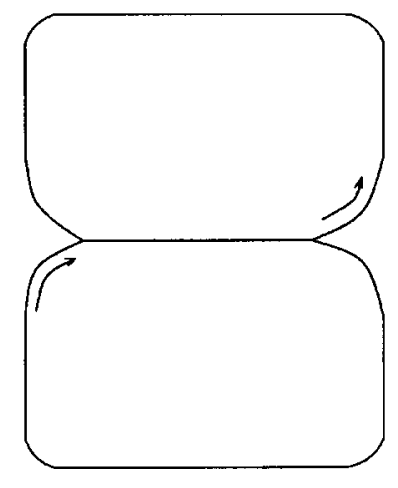

FIGURE 2

Proof. The map $f$ induces a map $\nu(f): D^{2} \rightarrow G_{7,5}$ via the normal bundle. Let us consider a fibration $\eta: E \rightarrow G_{7,5}$ with the Stiefel manifold $V_{5,2}$ as a fiber. Here $E$ is a subset of $G_{7,5} \times V_{7,2}$ defined in the following way: $L \times(a, b) \in E$ if and only if $a, b \in L$ and $\eta(L \times(a, b))=L$. First we choose a normal regular neighborhood $F^{1}$ of $\left.f\right|_{\partial D^{2}}$ of some radius $r$ and with Hopf invariant equal to 1 . The map $F^{1}$ induces a lifting $\beta: \partial D^{2} \rightarrow E$ of a map $\left.\nu(f)\right|_{\partial D^{2}}: \partial D^{2} \rightarrow G_{7,5}$. Since the fundamental group $\pi_{1}\left(V_{5,2}\right)$ is trivial, there is a lifting $\gamma: D^{2} \rightarrow E$ extending $\beta$. The map $\gamma$ defines a map $\Phi: D^{2} \times \mathbf{R}^{2} \rightarrow \mathbf{R}^{7}$ such that (1) $\left.\Phi\right|_{D^{2} \times\{0\}}=f,\left.(2) \Phi\right|_{\partial D^{2} \times D_{r_{0}}^{2}}=F^{1}$ and (3) for every $x \in$ Int $D^{2}$, the restriction $\left.\Phi\right|_{\{x\} \times \mathbf{R}^{2}}$ is an isometric imbedding into a normal plane $\nu(f)(x)$ at the point $f(x) \in \mathbf{R}^{7}$. In order to complete the proof we choose $r<r_{0}$ such that the restriction $F=\left.\Phi\right|_{D^{2} \times D_{r}^{2}}$ is a relative embedding.

We note that the following parameterized version of Lemma 7 is valid.

Lemma 8. Suppose that a sequence $f_{i}:\left(D^{2}, \partial D^{2}\right) \rightarrow\left(\mathbf{R}^{7}, \mathbf{R}^{3}\right)$ converges in $C^{2}$ metric to $f$. Then there exists $r>0$ and a sequence of maps $F_{i}:\left(D^{2} \times D_{r}^{2}, \partial D^{2} \times\right.$ $\left.D_{r}^{2}\right) \rightarrow\left(\mathbf{R}^{7}, \mathbf{R}^{3}\right)$ with the properties (1)-(3) of Lemma 6 for $f_{i}$ for every $i=$ $1,2, \ldots, \infty$, such that $F_{i}$ converges in $C^{2}$-metric to $F_{\infty}$.

Proof of Theorem 2. Consider the 3-sphere $S^{3}$ in $\mathbf{R}^{7}$ with a flat part in $\mathbf{R}^{3} \subset \mathbf{R}^{7}$. We define a sequence of embeddings $f_{i}:\left(D^{2}, \partial D\right) \rightarrow\left(\mathbf{R}^{7}, \mathbf{R}^{3}\right), C^{2}$-converging to a relative embedding $f_{\infty}$ with $f_{i}\left(\partial D^{2}\right) \subset S^{3}$ and $f_{i}\left(\right.$ Int $\left.D^{2}\right) \cap S^{3}=\emptyset$ for $i=1,2, \ldots, \infty$. Let $g: S^{1} \rightarrow \mathbf{R}^{3} \cap S^{3}$ be an immersion of the circle with the self-intersection along the interval as shown in Figure 2.

Let $f: D^{2} \rightarrow \mathbf{R}^{7}$ be an extension of $g$ to a relative $C^{2}$-embedding with $f\left(\operatorname{Int} D^{2}\right) \cap$ $\left(S^{3}\right)=\emptyset$. For every $i$ we realize the knot $K^{i}$ by a map $g_{i}: S^{1} \rightarrow \mathbf{R}^{3} \cap S^{3}$ such that the distance between $g$ and $g_{i}: S^{1} \rightarrow \mathbf{R}^{3}$ in $C^{2}$-metric is less than $1 / i$. For every $i$ there is an extension $f_{i}$ of $g_{i}$ which is $2 / i$-close to $f$ and moreover $f_{i}$ is an embedding. Apply Lemma 8 to the sequence $f_{i}$ to obtain $F_{i}, i=1,2, \ldots, \infty$. For every $i<\infty$ there exists a small number $\epsilon_{i}>0$ such that the restriction $R_{i}=\left.F_{i}\right|_{D^{2} \times \partial D_{\epsilon_{i}}^{2}}$ is an embedding. We define $\Sigma_{i}=S^{3}-\left(S^{1} \times D_{\epsilon_{i}}^{2}\right) \cup I m R_{i}$ for all $i$. It easy to check that $\Sigma_{i}$ is homeomorphic to $K_{1}^{i}$.

In order to complete the proof it is sufficient to find a common upper bound for $\alpha\left(\operatorname{Im} R_{i}\right)$. For large $i$ there is a rough estimate for $\alpha\left(\operatorname{Im} R_{i}\right)$ as $2 \pi \alpha\left(f\left(D^{2}\right)\right)$. That implies an upper bound for the sequence $\left\{\alpha\left(\operatorname{Im} R_{i}\right)\right\}$. Thus, Lemma 6 completes the proof. 
I am very thankful to V. I. Arnold and K. Johannson for conversations on the subject of this paper.

\section{REFERENCES}

[Ar] V. I. Arnold, Dynamics of complexity of intersections, Bol. Soc. Bras. Mat. 2:1 (1990), 1-10. MR 93c:58031

[Or] P. Orlik, Seifert manifolds (Lecture Notes in Math. 291), Springer-Verlag, Berlin, Heidelberg, New York, 1972. MR 54:13950

[D-N-F] B. A. Dubrovin, S. P. Novikov, A. T. Fomenko, Modern geometry (in Russian), vol. 1, Nauka, Moscow, 1979. MR 81f:53001

[H] M. W. Hirsch, Differential topology, Springer-Verlag, New York, Heidelberg, Berlin, 1976. MR 56:6669

[N-K] K. Nomizu, Sh. Kobayashi, Foundations of differential geometry,vol.2, Interscience publishers, New York, London, Sydney, 1969. MR 38:6501

[R] D. Rolfsen, Knots and Links, Publish or Perish, Berkeley, 1976. MR 58:24236; MR 95c:57018

[A-M] S. Akbulut and J. D. McCarty, Casson's invariant for oriented homology 3-spheres: an exposition, Univ. Press, Princeton, 1990. MR 90k:57017

Department of Mathematics, University of Florida, 358 Little Hall, Gainesville, FLORIDA 32611-8105

E-mail address: dranish@math.ufl.edu 